American Journal of Applied Sciences 9 (8): 1290-1293, 2012

ISSN 1546-9239

(C) 2012 Science Publications

\title{
Mathematical Optimum of the Audit Sample
}

\author{
Georgeta Ancuta Span and Irimie Emil Popa \\ Department of Accounting and Auditing, \\ Faculty of Economic Sciences and Business Administration, \\ Babes-Bolyai University Cluj-Napoca, Teodor Mihali 58-60, 400591, Cluj-Napoca, Cluj, Romania
}

\begin{abstract}
Problem statement: The primary objective of any audit mission is to obtain a high level of assurance on the fact that financial statements are prepared in accordance with a general financial reporting framework. Getting an absolute level of assurance is not possible due to the complexity and big number of transactions and operations found in practice. This research aims to identify the shortcomings of one of the sampling methods used by the Romania auditors (80/20 method) and it was proposed a mathematical function that leads to more appropriate results. Approach: Two examples were analyzed in order to highlight the importance of the proportion of operations in determining the mathematical optimum. The shortcoming of $80 / 20$ sampling method and the need for a mathematical optimum was shown through a general hypothetical case study. Results: Determining the proportion of small or big operations in an entity`s total transactions is not an arbitrarily decision, it has an optimal value depending on the accepted probability distribution of the operations value. Conclusion: Audit sampling depends on several parameters and has to fit a certain audit budget.
\end{abstract}

Key words: Accepted probability distribution, arbitrarily decision, certain audit budget, operations value, appropriate results, pareto method, audit sampling

\section{INTRODUCTION}

The starting point of a process is essential in conducting a mission and influences the expected results. In this respect, Boyer and Martin (2004) developed an optimization model which can be applied to determine an optimal audit contract. The study undertaken by them was based on Townsend model and it considered the importance of the agent type and the required audit strategy.

Dionne et al. (2009) develop an optimization model based on scoring technique. The analysis brings together audit and scoring in a verification procedure applied to insurance market. The proposed theory predicts that the optimal audit strategy is based on random checks, but the applications for insurance benefits must be audited with a probability of less than 1. Moreover, the optimal audit strategy should lead to minimization of the total costs in connection with fraud.

Another study on optimization methods in auditing with application in the public domain was led by Macho-Standler and Perez-Castrillo (1997). They demonstrated that audit should be organized taking into account the sources of the incomes to be certified. Moreover, the study discusses the necessary budget to achieve the auditor's objective. The lower the budget is, the smaller the check will be. At public level, the problem of income origin is determined by three factors, such as: tax evasion which varies from one activity to another, groups of taxpayers (especially those independent) which declare lower values than the real ones and the collection of revenues from the penalties paid by the taxpayers.

An important aspect of an audit, directly related to the procedures and strategies previously treated refers to the audit sample size. Researches regarding the optimization of this stage of the mission were made by Godfrey and Andrew (1982) who compared the size of a sample from a finite population and a sample size for infinite population, both of which being determined based on a Bayesian model. They determined the optimal sample size considering the prior distribution of the number of errors in the population, the certain level of reliability and the high limit of accuracy. The utility of the linear function proposed by them provides a trail in the audit sample size complexity with respect to the losses and costs of an audit.

The model proposed by Godfrey and Andrew was further developed by Fenwick and Trader (1986). In their study, based on the same Bayesian

Corresponding Author: Georgeta Ancuta Span, Department of Accounting and Auditing, Babes-Bolyai University ClujNapoca, Faculty of Economic Sciences and Business Administration, Teodor Mihali 58-60, 400591, Cluj-Napoca, Cluj, Romania 
model, the researchers showed that the optimal size of the sample could be determined by combining six sets of parameters which vary depending on the entity`s particularities.

Practice proved that is difficult to determine the amount and the level of errors from sampling, but, nevertheless, in order to optimize this stage of the engagement must also be taken into consideration the value of the possible sampling errors.

Setting the number and value of operations or transactions included in the audited sample is a difficult issue affecting the quality of results. At the beginning, auditors used nonstatistical sampling methods but recently, because of the economic environment complexity, sampling involves techniques borrowed from other areas such as statistics, computer science or mathematics.

The sampling methods used by the Romanian auditors meet the requirements of the professional standards on auditing, but the mathematical optimum it is not fulfilled in all the situations. The optimization principle refers to the effort minimization while the effects are maximized. In terms of sampling, optimization means auditing a large number of operations and transactions taking into consideration a specific budget.

One of the sampling methods used in Romania is the "80/20 method" known as Pareto method as well. This means there are audited $80 \%$ of the "big transactions" and $20 \%$ of the "small transactions" of an entity. The problem is how can be determined which operations are being considered to have a big value and which one can be included in the "small transactions" category.

Pareto technique requires editing a list of the most significant transactions descending ordered according to their importance, considering that $20 \%$ of them have an $80 \%$ effect on the whole population. In other words, this principle involves analyzing the overall population and selecting $20 \%$ of the most important operations/transactions. The selected operations will form the audit sample, with representative results for the entire population.

However, to avoid a strictly quantitative approach, it requires that the remaining $80 \%$ of the items to not be ignored. A distortion found on these operations, although not quantitatively significant may be important from a qualitative point of view and can change the way in which auditors interpret the final results.

For example, if an error has occurred because of fraud, even if it is not quantitatively significant, its nature might be serious and could influence the audit opinion.

Pareto method used in determining the audit sample has many shortcomings. First, it is difficult to choose $20 \%$ of those elements that are important enough to have an $80 \%$ effect on the entire population. It is difficult to quantify the importance of these transactions and their choice does not entail obtaining the optimal results. The purpose of our study is to determine an efficient function in order to establish the size of the audit sample so that the results to be optimal. In other words, in the limits of a certain budget, the auditors must select a certain number of operations and of a certain amount in order to obtain the optimal results.

Figure 1 shows the situation of the audited operations and their variation depending on their value (big operations and small operations).

There are considered the following notations:

- Total number of transactions of a particular type: $\mathrm{N}$

- Total number of audited transactions: $n$. This value is exogenous, being determined by the cost assigned to the audit mission

- The proportion of "small" operations in the total operations: $\lambda$

- Consequently, the number of "small operations" is: $\lambda \mathrm{N}$

- The proportion of "big operations" in total operations: $1-\lambda$

- Consequently, the number of "big operations" is: $(1-\lambda) \mathrm{N}$

- $\quad$ it is supposed a constant proportion of the wrong recorded operations compared with the value of the operation $\theta$

- The proportion of the "small operations" checked: Ps

- The number of "small operations" checked: Ps $\lambda \mathrm{N}$

- The number of "small operations" unchecked: (1Ps) $\lambda \mathrm{N}$

It is denoted with $\overline{\mathrm{x}}_{\mathrm{S}}$ the average of small operations and $\bar{x}_{B}$ the average of big operations. The mathematical expectation of the sum of the unverified transactions must be minimized (big + small operations):

$$
\left(1-p_{S}\right) \theta \lambda N \bar{x}_{S}+\left(1-p_{B}\right) \theta(1-\lambda) N \bar{x}_{B}
$$

But the number of small audited operations Ps $\lambda N$ plus the number of big audited operations $P_{B}$ (1$\lambda$ ) $\mathrm{N}$ must be equal to $\mathrm{n}$, which is a consequence of the audit budget. The mathematical expectation of the total value of the unverified wrong registered transactions $\left(1-p_{S}\right) \theta \lambda N \bar{x}_{S}+\left(1-p_{B}\right) \theta(1-\lambda) N \bar{x}_{B} \quad$ depends on: the number of the audited operations $n$, the distribution of the proportion $\theta$ which can be constant or variable depending on the value of the operations, the proportion of the operations considered to be small $\lambda$. 


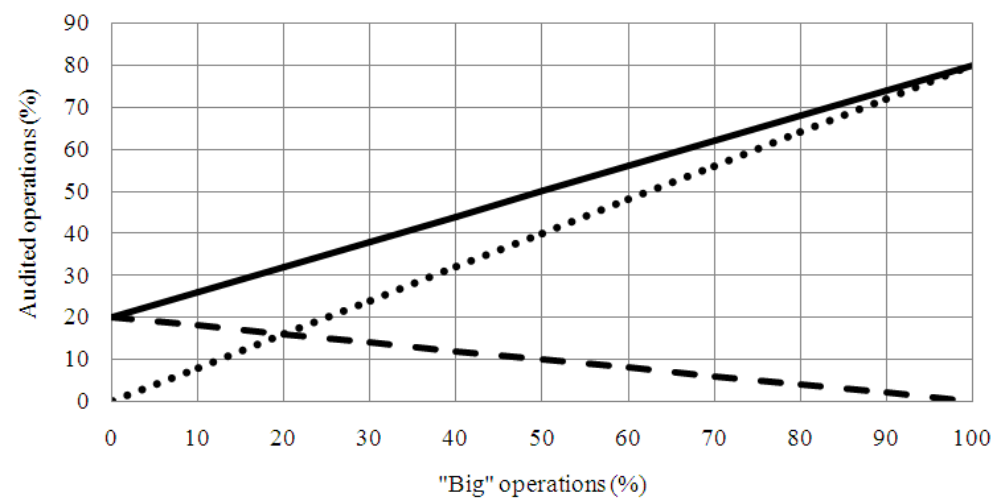

Fig. 1: The variation of the audited operations in relation to "big transactions"

It was demonstrated (Mare and Dragos, 2011) that $\lambda$ doesn't have to be arbitrarily determined, it has an optimal value depending on the accepted probability distribution of the operations value (the ratio between $\overline{\mathrm{x}}_{\mathrm{B}}$ and $\overline{\mathrm{x}}_{\mathrm{S}}$ ).

\section{MATERIALS AND METHODS}

The data used in the analyzes are the result of the authors` practical experience in auditing. The starting point of the method is represented by the average value of different parameters for a sample of audited firms. Using data from different practical cases are drawn some hypothetical general examples in order to show the need for a function to include the particularities of each entity. This approach could lead to more accurate results.

To better understand the ideas presented above there are proposed two hypothetical examples, as follows:

It is considered that the entity's "big transactions" represent $50 \%$ of the total transactions, according to Pareto technique, the auditor must verify $80 \%$ of this category of operations, more precisely $40 \%$ of the total operations $(0.8 * 0.5=40)$. The small operations will be checked in proportion of $20 \%$, more precisely $10 \%$ of the total operations $(0.2 * 0.5=10)$. Taking into account the situation, the auditor will audit $50 \%$ of the total operations ( $10 \%$ small operations $+40 \%$ big operations $=50 \%$ operations) .

On the other hand, if the entity has only $1 \%$ "big operations", the auditor must verify $0.8 \%$ of the total transactions $(0.8 * 0.01=0.8)$. The rest of $99 \%$ operations will be audited in a proportion of $20 \%$, which means $19.8 \%$ of the total operations $(0.2 * 0.99=$ 19.8). According to this second example, the auditor will verify $20.6 \%$ operations $(0.8 \%$ big operations + $19.8 \%$ small operations $=20.6 \%$ operations).

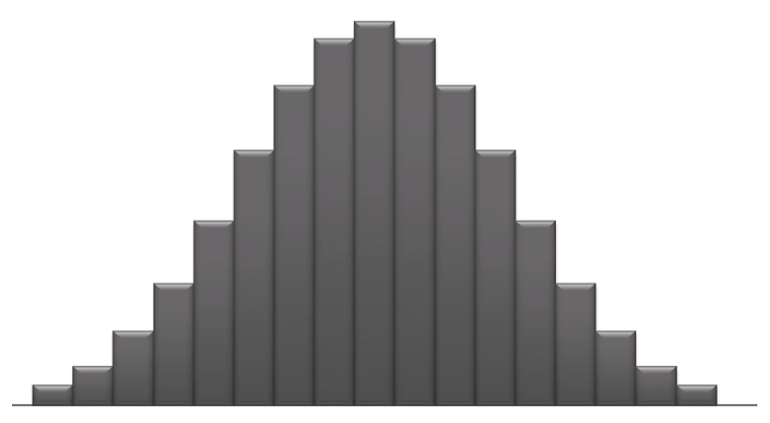

Fig. 2: Normal distribution of operations

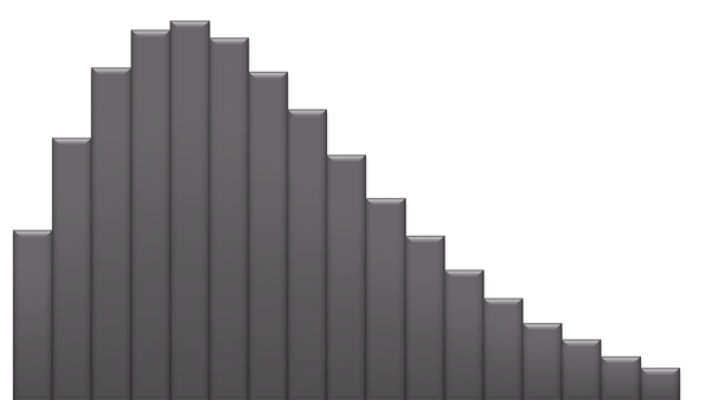

Fig. 3: Log-normal distribution of operations

Figure 1 shows the situation of the audited operations and their variation depending on their value (big operations and small operations).

It is considered a general case when all the operations have the same value. In general, according to the accounting practice, the distribution of values of a particular operations category is normal (Fig. 2) or lognormal (Fig. 3). A study that reflects the distribution of the operations within an entity was conducted by Mare and Dragos (2011). 
When using Pareto method, the auditor is tempted to consider as few operations as possible being part of the "big operations" category, in order to reduce the level of his study and to fit the budget.

\section{RESULTS}

The "80/20" method used in auditing practice is purely indicative. The mathematical optimum of the operations number and structure depends on several parameters. Estimating these parameters requires an additional cost. It should be compare on empirical data this cost to the benefit of using the mathematical optimum in sampling in relation to the professional sampling methodologies.

The optimization model developed by us is a simple model under the restriction of several assumptions:

- Consideration of a single type of operations and a single constant percentage of the wrong recorded operations compared to the operations' values

- Consideration of a constant cost of checking an operation compared to the operation value

\section{DISCUSSION}

Further research will focus on the development of this model under the flexibility of these assumptions. We did, however, demonstrate that the selection of the operations that must be audited depends on several parameters such as: The value distribution function of a single type operations, the percentage of wrong recorded transactions and the number of operations to be audited (budget restriction).

\section{CONCLUSION}

The audit methodology for determining the audit sample based on the professional standards or on the judgment uses the average values of the elements to be audited. According to this approach, there can be important differences from one entity to another involving overestimated or underestimated audits compared with the mathematical optimum. These facts are reflected either in additional costs or in reducing the quality and precision of the final results.
In order to estimate the value of these deviations it is required an appropriate estimation of the parameters considered for a sample of audited companies. Thus, with the help of the econometric tools we can estimate the deviations of costs and precisions guaranteed by the audit results.

\section{ACKNOWLEDGMENT}

Invest in people! Project co-financed by the European Social Fund, Sectoral Operational Programme Human Resources Development 20072013, POSDRU 64076, Babeş-Bolyai University, ClujNapoca, Romania

\section{REFERENCES}

Boyer, M. and G.P. Martin, 2004. Optimal audit policies with correlated types. Econ. Theory, 24: 325-334.

Dionne, G., E. Giuliano and P. Picard, 2009. Optimal auditing with scoring: Theory and application to insurance fraud. Manage. Sci., 55: 58-70. DOI: $10.1287 / \mathrm{mnsc} .1080 .0905$

Fenwick, H.H. and T.L. Ramona, 1986. A note on optimal sample sizes in compliance tests using a formal Bayesian decision-theoretic approach for finite and infinite populations. J. Accou. Res., 24: 394-399.

Godfrey, J.T. and R.W. Andrews, 1982. A finite population Bayesian model for compliance testing. J. Accou. Res., 20: 304-315.

Macho-Standler, I. and J.D. Perez-Castrillo, 1997. Optimal auditing with heterogeneous income. Int. Econ. Rev., 38: 951-968.

Mare, C. and C. Dragos, 2011. Small operations versus big operations-mathematical modeling of optimality in audit sample. Int. Res. J. Applied Finance, 2: 1362-1368 